\title{
Reviewer acknowledgement 2015
}

\author{
Jay Duker ${ }^{1 *}$ and Eduardo Rodrigues ${ }^{2}$
}

\begin{abstract}
The Editors of International Journal of Retina and Vitreous would like to thank all of our reviewers, both external and Editorial Board Members, who have contributed to the journal in Volume 1 (2015) and whose valuable support is fundamental to the success of the journal.
\end{abstract}

\section{Acácio Muralha}

Brazil

Aderbal de Albuquerque Alves Brazil

\section{Airton Kronbauer \\ Brazil}

Alessandro Daré
Brazil

Alex Hunyor

Australia

Alexandre Ventura

Brazil

Alice Degoumois

Brazil

\section{Anat Loewenstein}

Israel

Andre Gomes
Brazil

Andre Romano

Brazil

Antonio Marcelo Barbante Casella Brazil

\section{Arnaldo Furman Bordon \\ Brazil}

Arturo Alezzandrini

Argentina

\section{Baruch Kuppermann}

United States of America

\section{Caio Regatieri}

United States of America

\section{Caio Augusto Scocco}

Brazil

Carl Regillo

United States of America

\section{Carlos Veloso}

Brazil

\section{Carol Shields}

United States of America

\section{Carsten Meyer}

switzerland

\section{Chen Hao-Yu \\ China}

Christopher Brady

United States of America

\section{Daniel Lavinsky}

Brazil

Daniela Ferrara

United States of America

David Boyer

United States of America

David Leonardo Isaac

Brazil

Debra Goldstein

United States of America

Eduardo Souza

Brazil

Eduardo Novais

Brazil

Eduardo Cunha de Souza

Brazil

Emmerson Badaró

Brazil

Enzo Fulco

Brazil

Evandro Lucena

Brazil

${ }^{*}$ Correspondence: jduker@tuftsmedicalcenter.org

${ }^{1}$ Tufts University School of Medicine, Boston, USA

Full list of author information is available at the end of the article 
Fang Wang

China

Fernando Penha

Brazil

Fernando Arevalo

Saudi Arabia

Fernando Jose de Novelli

Brazil

Focke Ziemsen

Germany

Francesco Rodriguez
Colombia

Francisco Max D’Amico

Brazil

Gregory Lee

United States of America

Helio Francisco Shiroman

Brazil

Hidenori Takahashi

Japan

Irena Tsui

United States of America

Jaime Roizenblatt

Brazil

James Augsburger

United States of America

Jay Chhablani

India

João Dias

Brazil

João Luiz Lobo Ferreira

Brazil

John Helal Junior

Brazil

Jorge Mitre

Brazil

Jorge Carlos Pessoa Rocha

Brazil
Jose PUlido

United States of America

Junior Kuczmainski

Brazil

Kleyton Barella

Brazil

Kouros Panagiotis

Switzerland

Koushik Tripathy

Brazil

Laurentino Biccas

Brazil

Leandro Cabral Zacharias

Brazil

Leonardo Cunha Castro

Brazil

Lihteh Wu

Costa Rica

Luiz Lima

Brazil

Luiz Henrique Lima

Brazil

M Chéour

France

Magno Ferreira

Brazil

Makoto Inoue

Japan

Marcin Stopa

Poland

Marcio Nehemy

Brazil

Mariana Matioli

Brazil

Mário Nóbrega

Brazil

Mary E Hartnett

United States of America
Mathew MacCumber

United States of America

Matus Rehak

Germany

Mauricio Maia

Brazil

Mauro Goldbaum

Brazil

Michael Singer

United States of America

Michel Farah

Brazil

Minoru Furuta

Japan

Muhammad Moazam Fraz

Pakistan

Murat Hasanreisoglu

Turkey

Nicolas Feltgen

Germany

Noemi Lois

United Kingdom

Ossewaarde-van Norel

Netherlands

Paulina Haas

Germany

Paulo Augusto de Arruda Mello Filho

Brazil

Peter Traine

Switzerland

Priscilla Ballalai

Brazil

Quan Nguyen

United States of America

Rafael Caiado

Brazil

Raja Narayanan

India 


\section{Rajendra Apte}

United States of America

\section{Ranjana Mathur}

Singapore

\section{Raul Vianna}

Brazil

Rodrigo Meirelles

Brazil

Rodrigo Lira

Brazil

\section{Sandeep Saxena \\ India}

Serdar Bilici

Turkey

Sérgio Pimentel

Brazil

Szilard Kiss

United States of America

Taiichi Hikichi

Japan

\section{Tatsuhiko Sato}

Japan

Thomas Theelen

Netherlands

\author{
Vincent Daien \\ France
}

\section{Vinicius Kniggendorf}

Brazil

\section{Vinícius Saraiva}

Brazil

Zihret Abazi

Serbia and Montenegro

\footnotetext{
Author details

${ }^{1}$ Tufts University School of Medicine, Boston, USA.

${ }^{2}$ Federal University of São Paulo, São Paulo, Brazil.
}

Received: 27 January 2016 Accepted: 27 January 2016

Published online: 16 February 2016

Submit your next manuscript to BioMed Central and we will help you at every step:

- We accept pre-submission inquiries

- Our selector tool helps you to find the most relevant journal

- We provide round the clock customer support

- Convenient online submission

- Thorough peer review

- Inclusion in PubMed and all major indexing services

- Maximum visibility for your research

Submit your manuscript at www.biomedcentral.com/submit 